\title{
Superstring Phenomenology in Light of LEP, KamLAND and WMAP
}

\author{
Alon E. Faraggi ${ }^{1}$ \\ Theoretical Physics Department, University of Oxford, Oxford OX1 3NP, UK
}

\begin{abstract}
The experimental data of the past decade suggests that the quantum gravity vacuum should possess two key ingredients. The existence of three generations and their embedding in $S O(10)$ representations. The $Z_{2} \times Z_{2}$ orbifold of the heterotic string provides examples of vacua that accommodate these properties. The utilization of string dualities to explore these models is discussed. Classification of the $Z_{2} \times Z_{2}$ orbifold with geometric shifts on complex manifolds demonstrates that three generations are not obtained solely with symmetric shifts on complex tori, but necessarily utilize an asymmetric shift or a nonperturbative breaking of the GUT gauge group. The issue of mass and mixing spectrum in the neutrino versus the quark sector is examined.
\end{abstract}

\section{Introduction}

\subsection{Experimental guidelines}

The passing decade provided remarkable amount of high quality experimental data, that deeply affects our perception of physical reality. The collider experiments, in particular the LEP collaboration at CERN 11, confirmed the validity of the Standard Model to a degree precedented only by quantum electrodynamics. The precision measurements at LEP also yielded one of the vital experimental clues for physics beyond the Standard Model. By restricting the number of light left-handed neutrinos below the $Z$-threshold to $N_{\nu} \sim 3$, LEP has constrained the number of light chiral generations to be three and only three! This observation is one of the vital clues in the quest for the fundamental unification of the matter and interactions. In the course of the previous decade the impressive output of the LEP collaboration continued relentlessly, providing for the first time solid confirmation of the three and four gauge boson vertices, hence confirming the non-Abelian character of the electroweak interactions. The wealth of experimental data from LEP is a triumph of scientific indulgence.

In the passing five years the neutrino observatories produced revealing new data on the neutrino sector of the Standard Model [234, with profound implications on its fundamental origins. This new data represents the pinnacle of accumulative and steady progress in the experimental detection of neutrinos over the past fifty years. For the first time it provides conclusive evidence that the neutrinos are massive and that the Standard Model spectrum is augmented by the right-handed neutrinos. While this eventuality has been anticipated since the early seventies [5], one should note the profound difference between naive theoretical expectations versus the experimentally confirmed observations. 
In the past year the WMAP collaboration [6] provided unprecedented accurate measurements of the cosmic microwave background radiation. The new data impose further constraints on the range of allowed neutrino masses. But, perhaps more importantly, it brings cosmological observations closer to providing valuable quantitative data that may prove to be revealing on the physical properties of the universe. In the least it provides further accurate data on the energy composition of the universe. Perhaps more intriguing is the, yet statistically insignificant, indications of correlations at large angles. If proved correct these will have unprecedented implications on the structure of the universe. In particular on its potential realization in the framework of string theory.

Lastly, the forthcoming decade promises to be even grander, with AUGER, PLANCK, LHC, The Linear Collider (TLC), and other experiments.

\subsection{Theoretical considerations}

The augmentation of the Standard Model by the right-handed neutrinos has profound implications on its fundamental origin. It provides strong support for the embedding of the Standard Model spectrum, generation by generation, in spinorial 16 representation of $S O(10)$. Furthermore, the neutrino data from cosmic and reactor experiments indicate surprising new structures that go beyond the conventional GUT expectations. The observational data from collider facilities and cosmic observatories indicates two pivotal ingredients in the quest to understand the fundamental origins of the Standard Model. The existence of three generations and their embedding, generation by generation, in spinorial 16 representations of $S O(10)$. In addition to the gauge sector the Standard Model data consists of the flavor sector. While the gauge observables find their natural origins in Grand Unified Theories, the flavor observables do not. This implies that fundamental understanding of the flavor parameters must arise from a theory that unifies the gauge and gravitational interactions. String theory provides a consistent contemporary framework for perturbative quantum gravity. As such string theory produces viable machinery to study how a fundamental theory of quantum gravity may determine the Standard Model parameters.

Additionally the consistency of string theory requires some additional structures beyond the Standard Model. These arise in the form of extra dimensions, supersymmetry and additional matter and gauge states beyond the SM spectrum. The question, however, is whether these additional structures yield any experimental imprints that may be observed. Since the main collider experiments in the forthcoming decades will target the physics at the electroweak scale, and the associated Higgs mass generation mechanism, it is hoped that at least some of these structures will be accessible in such experiments. These may come in the form of low scale supersymmetry, or large extra dimensions that arise in some limits of the underlying string theory. Additionally, proton decay experiments and cosmic observatories may provide experimental probes to the physics at larger scales.

The utility of string theory in my view is to study how the parameters of the Standard Model may arise from a theory of quantum gravity. In the past 
few years a new view of string theories has been developed [7. In the framework of M-theory the five different string theories in ten dimensions, together with eleven dimensional supergravity are seen to be limits of a more fundamental theory, traditionally dubbed M-theory. We should therefore question how this new picture affects the utilization of string theory toward phenomenological studies. In this respect we should regard the perturbative string limits as effective limits, non of which can fully characterize the true vacuum, which should possess some non-perturbative realization. The new picture of M-theory suggests that the effective limits may at best probe some features of the non-perturbative vacuum, and that different limits may be instrumental to extract different properties. For example, the $S O(10)$ embedding of the Standard Model spectrum can only be seen in the heterotic limit because this is the only limit that gives rise to the chiral 16 of $S O(10)$ in the effective low energy field theory. On the other hand, in the perturbative heterotic-string limit the dilaton, whose VEV governs the string gauge and gravitational couplings, has a run-away potential and cannot be stabilized at a finite value. However, we should regard the heterotic limit as the zero coupling expansion of the more basic theory. With our present understanding of string theories in the context of their M-theory embedding it is clear that we should not in fact expect the dilaton to be stabilized in the heterotic limit. In order to stabilize the dilaton we have to move away from the zero coupling expansion, or to move away from the perturbative heterotic-string limit. The existence of the classical eleven dimensional limit in which the dilaton is interpreted as the moduli of the eleventh dimension lends credence to this general expectation. Thus, the issue of dilaton stabilization may be more accessible, even if not yet fully resolved, in other limits of the underlying theory, rather than in the perturbative heterotic string limit.

The new M-theory picture therefore suggests the following approach toward utilization of string theory for phenomenological studies. Suppose that in some effective limit we are able to identify a string vacuum that exhibits viable phenomenological characteristics. This would entail identifying a particular class of string compactifications on which the effective string theory is compactified. The new picture of M-theory then suggests that additional information on the properties of the non-perturbative vacuum may be gleaned by compactifying other string limits on the same class of manifolds.

In this talk I will discuss the efforts to develop such an approach undertaken by the string phenomenology group at Oxford. Additionally I will discuss recent work the geometrical correspondence of the three generation free fermionic models. These models explain the origin of the three generations as arising from the three twisted sectors of the $Z_{2} \times Z_{2}$ orbifold compactification. Hence the improved understanding of the geometrical correspondence of the free fermionic models aims to develop a geometrical explanation for the origin of the three generations. I discuss the potential implications of the large neutrino mixing angles for string and beyond the Standard Model phenomenology, and recent efforts aimed at developing the tools to utilize the forthcoming Ultra High Energy Cos- 
mic Ray (UHECR) experiments to probe physics beyond the Standard Model and string theory.

\section{High versus low}

To illustrate the approach toward string phenomenology advocated in this paper we have to make some judicial assumptions in regard to the physics beyond the Standard Model. There are basically two orthogonal directions that may be pursued. The first assumes that the Standard Model remains perturbative up to a very high scale, and that the ultra-violet cut-off, set by quantum gravity, is at the Planck scale. The second essentially assume that the Standard Model breaks down already not far beyond the electroweak scale and that the ultra-violet nonperturbative cut-off is at the $\mathrm{TeV}$, or multi- $\mathrm{TeV}$ scale. In this paper the first possibility is pursued. Aside from the elegant $S O(10)$ embedding of the Standard Model spectrum, this case is also supported by the viability of logarithmic running of the Standard Model gauge and matter sectors parameters, whereas the scalar sector requires the introduction of supersymmetry. Proton longevity and suppression of left-handed neutrino masses lends additional support to this picture.

Thus, the basic properties that we would like our string vacuum to possess are the existence of three chiral generations and their embedding in $S O(10)$ representations. A class of string models that yield these properties are the three generation free fermionic models. The structure of these models and related phenomenological studies have been amply discussed in the past and therefore in this paper only a brief discussion will be given.

\section{Dualities}

String theory exhibits various forms of dualities, i.e. relation between different theories at large and small radii of the compactified manifold and at strong and weak coupling. The first type is the T-duality $[8]$. Consider a point particle moving on a compactified dimension $X$, which obeys the condition $X \sim X+2 \pi R m$. Single valuedness of the wave function of the point particle $\Psi \sim \operatorname{Exp}(i P X)$ implies that the momenta in the compact direction is quantized $P=\frac{m}{R}$ with $m \in Z$. Now consider a string moving in the compactified direction. In this case the string can wrap around the compactified dimension and produce stable winding modes. Hence the left and right-moving momenta in the case of the closed string are given by

$$
P_{L, R}=\frac{m}{R} \pm \frac{n R}{\alpha^{\prime}}
$$

and the mass of the string states is given by

$$
\operatorname{mass}^{2}=\left(\frac{n}{R}\right)^{2}+\left(\frac{m R}{\alpha^{\prime}}\right)^{2}
$$


this is invariant under exchange of large and small radius together with the exchange of winding and momentum modes, i.e.

$$
\frac{1}{R} \leftrightarrow \frac{R}{\alpha^{\prime}} \quad \text { with } \quad m \leftrightarrow n
$$

and is an exact symmetry in string perturbation theory. Furthermore, there exist the self-dual point,

$$
R=\frac{\alpha^{\prime}}{R},
$$

which is the symmetry point under T-duality. In the case of the supersymmetric string on a compactified coordinate the $\mathrm{T}$-duality operation interchanges

$$
\begin{array}{lll}
\text { type IIA } & \Leftrightarrow & \text { type IIB } \\
\text { Heterotic } S O(32) & \Leftrightarrow & \text { Heterotic } E_{8} \times E_{8}
\end{array}
$$

Now, all this is of course well known since the late 80's. However, the following point is not well appreciated. It is also well known that for specific values of its radius, the compactified coordinate can be realized as specific rational conformal field theories propagating on the string world-sheet. In particular, there exist such a value for which a compactified coordinate can be represented in terms of two free Majorana-Weyl fermions. It so happens that, in some normalization, the self-dual point is at $R=1 / \sqrt{2}$ whereas the free fermionic point is at $R=1$. Hence, the two points do not overlap and the free fermionic point does not coincide with the self-dual point [9]. However, this is merely an artifact of the fact that we have been talking here about bosonic string. In the case of the supersymmetric string the two points, in fact, do coincide. This is a remarkable observation for the following reason. While we do not yet know at what value the compactified coordinate are fixed, naively we would expect that they are stabilized around a symmetry point or at infinity. The self-dual point under Tduality is precisely such a symmetry point. Hence, near the self-dual point, which is the symmetry point under T-duality and around which we may expect that the compactified dimensions stabilize, we can represent the compact dimension as a pair of free Majorana-Weyl fermions propagating on the string worldsheet. Of course, the real picture may be much more complicated. But a a first approximation this is the naive expectation, based on the symmetry properties of string theory.

T-duality is perturbative and exhibit itself in the exchange of the spectrum and the superpotential. Thus, it can be checked order by order in perturbation theory. In the past decade significant progress in understanding duality symmetries which are nonperturbative, i.e. that exchange weak with the strong coupling, has been achieved. The starting point in this program was the SeibergWitten solution of $N=2$ supersymmetric pure $S U(2)$ gauge theory [10]. In the supersymmetric theory the gauge coupling is extended to a complex parameter $\tau=\theta / 2 \pi+i 4 \pi / g^{2}$ where $\theta$ is the axial coupling and $g$ is the field strength coupling. The strong-weak duality extends to a $S L(2, Z)$ transformations of the 
parameter $\tau$. In the Seiberg-Witten solution the exact vacuum structure of the theory is parameterized in terms of a genus one Riemann surface.

In string theory we have naively a similar situation. The gauge coupling is fixed by the VEV of the dilaton field. The dilaton field, combined with the spacetime components of the antisymmetric tensor field forms a modular parameter. In M-theory [7] this complex field is identified with the moduli field of a new dimension and hence the $S L(2, Z)$ symmetry of this moduli field translates into a duality which exchanges strong and weak coupling 1112. The different string limits are related under the strong-weak coupling exchange, and by $\mathrm{T}$-duality after compactification to a lower dimension. The question that we examine in the work reported here is how to utilize this novel understanding of string theory for phenomenological studies.

\section{$4 \quad$ Realistic string models}

As discussed above the key properties that we would like our string vacuum to possess are the existence of three generations and their embedding in $S O(10)$ representations. The only effective string limit that preserves the $S O(10)$ embedding is the heterotic limit, because this is the only limit that produces the chiral 16 representation of $S O(10)$ in the perturbative spectrum. To build realistic models we compactify the ten dimensional heterotic string to the heterotic string in four dimensions. This is achieved by choosing a six dimensional manifold on which the string theory is compactified. Typically, the six dimensional internal manifolds can be represented as toroidal orbifolds. A $D$-dimensional tori is represented in terms of a $D$-dimensional Euclidean space, modded by a lattice translation. The orbifold is in turn obtained by moding the $D$-dimensional torus by an internal symmetry. Thus, for example, the circle is one dimensional Euclidean space modded by $2 \pi R$ identification under translation, and its $Z_{2}$ orbifold, which is a line segment with two fixed points, is obtained by identifying points by reflection across the real axis.

The $Z_{2}$ orbifold plays a crucial role in the discussion to follow. The three generation free fermionic models correspond to $Z_{2} \times Z_{2}$ orbifold of a six dimensional torus. The first $Z_{2}$ acts on the first four coordinates, whereas the second acts on the last four. The $Z_{2} \times Z_{2}$ orbifold models, through their realization in the free fermionic formulation, produce three generation models with $S O(10)$ embedding. Furthermore, the structure of the $Z_{2} \times Z_{2}$ orbifold naturally correlates the existence of three generation in nature with the underlying geometry. This arises due to the fact that each the $Z_{2} \times Z_{2}$ orbifold has exactly three twisted sectors. Each of the light chiral generations then arises from a distinct twisted sector. Hence, in these models the existence of three generations in nature is seen to arise due to the fact that we are dividing a six dimensional compactified

manifold into factors of 2 . In simplified terms, three generations is an artifact of

$$
\frac{6}{2}=1+1+1
$$


One may further ask whether there is a reason that the $Z_{2}$ orbifold would be preferred versus higher orbifolds. Previously we argued that the free fermionic point coincides with the self-dual point under $T$-duality, which is where we would naively expect the compactified dimensions to stabilize. The special property of the $Z_{2}$ orbifold that sets it apart from higher orbifolds, is the fact that the $Z_{2}$ orbifold is the only one that acts on the coordinates as real coordinates, rather than complex coordinates. Whether this property plays a role in the string vacuum selection is yet to be understood.

\section{$5 \quad$ Free fermionic model building}

The three generation $Z_{2} \times Z_{2}$ orbifold models were studied in the free fermionic formulation 14. These models were reviewed in the past in these conference series [13, and I therefore give here only a brief summary. The models are constructed in terms of a set of boundary condition basis vectors that define the transformation properties of the 20 left-moving and 44 right-moving real fermions around the noncontractible loops of the one-loop vacuum to vacuum amplitude.

The first five basis vectors of the realistic free fermionic models consist of the NAHE set [15]. The gauge group after the NAHE set is $S O(10) \times E_{8} \times S O(6)^{3}$ with $N=1$ space-time supersymmetry, and 48 spinorial 16 of $S O(10)$, sixteen from each sector $b_{1}, b_{2}$ and $b_{3}$. The three sectors $b_{1}, b_{2}$ and $b_{3}$ are the three twisted sectors of the corresponding $Z_{2} \times Z_{2}$ orbifold compactification. The $Z_{2} \times Z_{2}$ orbifold is special precisely because of the existence of three twisted sectors, with a permutation symmetry with respect to the horizontal $S O(6)^{3}$ charges.

The NAHE set is common to a large class of three generation free fermionic models. The construction proceeds by adding to the NAHE set three additional boundary condition basis vectors which break $S O(10)$ to one of its subgroups: $S U(5) \times U(1)$ [16], $S O(6) \times S O(4)$ [1], $S U(3) \times S U(2) \times U(1)^{2}[18192021$, or $S U(3) \times U(1) \times S O(4)$ 22]. At the same time the number of generations is reduced to three, one from each of the sectors $b_{1}, b_{2}$ and $b_{3}$. The various three generation models differ in their detailed phenomenological properties. However, many of their characteristics can be traced back to the underlying NAHE set structure. One such important property to note is the fact that as the generations are obtained from the three twisted sectors $b_{1}, b_{2}$ and $b_{3}$, they automatically possess the Standard $S O(10)$ embedding. Consequently the weak hypercharge, which arises as the usual combination $U(1)_{Y}=1 / 2 U(1)_{B-L}+U(1)_{T_{3_{R}}}$, has the standard $S O(10)$ embedding.

The massless spectrum of the realistic free fermionic models then generically contains three generations from the three twisted sectors $b_{1}, b_{2}$ and $b_{3}$, which are charged under the horizontal symmetries. The Higgs spectrum consists of three pairs of electroweak doublets from the Neveu-Schwarz sector plus possibly additional one or two pairs from a combination of the two basis vectors which extend the NAHE set. Additionally the models contain a number of $S O(10)$ 
singlets which are charged under the horizontal symmetries and a number of exotic states.

Exotic states arise from the basis vectors which extend the NAHE set and break the $S O(10)$ symmetry 23. Consequently, they carry either fractional $U(1)_{Y}$ or $U(1)_{Z^{\prime}}$ charge. Such states are generic in superstring models and impose severe constraints on their validity. In some cases the exotic fractionally charged states cannot decouple from the massless spectrum, and their presence invalidates otherwise viable models 24 25]. In the NAHE based models the fractionally charged states always appear in vector-like representations. Therefore, in general mass terms are generated from renormalizable or nonrenormalizable terms in the superpotential. However, the mass terms which arise from nonrenormalizable terms will in general be suppressed, in which case the fractionally charged states may have intermediate scale masses. The analysis of ref. [21] demonstrated the existence of free fermionic models with solely the MSSM spectrum in the low energy effective field theory of the Standard Model charged matter. In general, unlike the "standard" spectrum, the "exotic" spectrum is highly model dependent.

\section{Phenomenological studies of free fermionic models}

I summarize here some of the highlights of the phenomenological studies of the free fermionic models. This demonstrates that the free fermionic string models indeed provide the arena for exploring many the questions relevant for the phenomenology of the Standard Model and Unification. The lesson that should be extracted is that the underlying structure of these models, generated by the NAHE set, produces the right features for obtaining realistic phenomenology. It provides further evidence for the assertion that the true string vacuum is connected to the $Z_{2} \times Z_{2}$ orbifold in the vicinity of the free fermionic point in the Narain moduli space. Many of the important issues relating to the phenomenology of the Standard Model and supersymmetric unification have been discussed in the past in several prototype free fermionic heterotic string models. These studies have been reviewed in the past and I refer to the original literature and additional review references 2613 . These include among others: top quark mass prediction 20, several years prior to the actual observation by the CDF/D0 collaborations 27]; generations mass hierarchy [28; CKM mixing 29]; superstring see-saw mechanism [30; Gauge coupling unification [31; Proton stability [32]; supersymmetry breaking and squark degeneracy 3334. Additionally, it was demonstrated in ref. 21] that at low energies the model of ref. [18, which may be viewed as a prototype example of a realistic free fermionic model, produces in the observable sector solely the MSSM charged spectrum. Therefore, the model of ref. [18, supplemented with the flat F and D solutions of ref. 21], provides the first examples in the literature of a string model with solely the MSSM charged spectrum below the string scale. Thus, for the first time it provides an example of a long-sought Minimal Superstring Standard Model! We have therefore identified a neighborhood in string moduli space which is potentially relevant for low 
energy phenomenology. While we can suggest arguments, based on target-space duality considerations why this neighborhood may be selected, we cannot credibly argue that similar results cannot be obtained in other regions of the string moduli space. Nevertheless, the results summarized here provide the justification for further explorations of the free fermionic models. Furthermore, they provide motivation to study these models in the nonperturbative context of $\mathrm{M}$-theory. In this context the basis for our studies is the connection of the free fermionic models with the $Z_{2} \times Z_{2}$ orbifold, to which I turn in section 7

I would like to emphasize that it is not suggested that any of the realistic free fermionic models is the true vacuum of our world. Indeed such a claim would be folly. Each of the phenomenological free fermionic models has its shortcomings, that if time and space would have allowed could have been detailed. While in principle the phenomenology of each of these models may be improved by further detailed analysis of supersymmetric flat directions, it is not necessarily the most interesting avenue for exploration. The aim of the studies outlined above is to demonstrate that all of the major issues, pertaining to the phenomenology of the Standard Model and unification, can in principle be addressed in the framework of the free fermionic models, rather than to find the explicit solution that accommodates all of these requirements simultaneously. The reason being that even within this space of solutions there is till a vast number of possibilities, and we lack the guide to select the most promising one. What is being proposed is that these phenomenological studies suggest that the true string vacuum may share some of the gross structure of the free fermionic models. Namely, it will possess the structure of the $Z_{2} \times Z_{2}$ orbifold in the vicinity of the free fermionic point in the Narain moduli space. This perspective provides the motivation for the continued interest in the detailed study of this gross structure, and specifically in the framework of M-theory, as discussed below.

The free fermionic models also serve as a laboratory to study possible signatures beyond the Standard Model. Perhaps most fascinating among those is the existence of exotic matter states 3523 that can lead to to experimental signatures in the form of energetic neutrinos from the sun [36, or in the form of candidates for dark matter and top-down UHECR scenarios [37. The later is particularly exciting due to the forthcoming Pierre Auger and EUSO experiments that will provide more statistics on UHECR.

\section{Correspondence with $Z_{2} \times Z_{2}$ orbifold}

The key property of the free fermionic models that is sought in the attempts to investigate these models in the nonperturbative framework of $\mathrm{M}$-theory is their relation to the $Z_{2} \times Z_{2}$ orbifold. In the fermionic language the models are defined in terms of the boundary condition basis vectors. Extending the NAHE set with the additional basis vector $\xi_{2}$, we can regenerate the model defined by this set by using instead the set $\left\{\mathbf{1}, S, \xi_{1}, \xi_{2}, b_{1}, b_{2}\right\}$. Here the vacuum produced by the four basis vectors $\left\{\mathbf{1}, S, \xi_{1}, \xi_{2}\right\}$ is an $N=4$ supersymmetric toroidal compactification with $S O(12) \times E_{8} \times E_{8}$ right-moving gauge group. Adding the 
two basis vectors $b_{1}$ and $b_{2}$ correspond to the $Z_{2} \times Z_{2}$ action and produces a vacuum with $S O(4)^{3} \times E_{6} \times U(1)^{2} \times E_{8}$ and 27 generations plus 3 antigenerations of the 27 representation of $E_{6}$. The same model is obtained in the bosonic language by specifying the VEVs of the background fields that produce the enhanced $S O(12)$ lattice and moding by the $Z_{2} \times Z_{2}$ orbifold projections 38. The resulting manifold has $\left(h_{1,1}, h_{2,1}\right)=(27,3)$ and is referred to as $X_{2}$.

The $Z_{2} \times Z_{2}$ orbifold at the free fermionic point in the Narain moduli space hence produces a vacuum with a net number of 24 generations. However, the $Z_{2} \times Z_{2}$ orbifold at a generic point in the moduli space produces a model with $\left(h_{1,1}, h_{2,1}\right)=(51,3)$ or a net number of 48 generations. I denote this manifold as $X_{1}$. Hence, there is a discrepancy by a factor of 2 between the two models. This discrepancy is in fact crucial both from the point of view of pursuing the $\mathrm{M}$-embedding of these models as well as trying to understand the origin of the three generations. Below we expand on these aspects.

For our purpose here it is important to observe that the two manifolds, $X_{1}$ and $X_{2}$, may be connected by adding a freely acting twist or shift. Let us first start with the compactified $T_{2}^{1} \times T_{2}^{2} \times T_{2}^{3}$ torus parameterized by three complex coordinates $z_{1}, z_{2}$ and $z_{3}$, with the identification

$$
z_{i}=z_{i}+1, \quad z_{i}=z_{i}+\tau_{i}
$$

where $\tau$ is the complex parameter of each $T_{2}$ torus. With the $Z_{2}$ identification $z_{i} \rightarrow-z_{i}$, a single torus has four fixed points at $z_{i}=\left\{0, \frac{1}{2}, \frac{1}{2} \tau, \frac{1}{2}(1+\tau)\right\}$. With the two $Z_{2}$ twists

$$
\begin{aligned}
& \alpha:\left(z_{1}, z_{2}, z_{3}\right) \rightarrow\left(-z_{1},-z_{2}, z_{3}\right), \\
& \beta:\left(z_{1}, z_{2}, z_{3}\right) \rightarrow\left(z_{1},-z_{2},-z_{3}\right),
\end{aligned}
$$

there are three twisted sectors in this model, $\alpha, \beta$ and $\alpha \beta=\alpha \cdot \beta$, each producing 16 fixed tori, for a total of 48 . Adding to the model generated by the $Z_{2} \times Z_{2}$ twist in (4), the additional freely acting shift

$$
\gamma:\left(z_{1}, z_{2}, z_{3}\right) \rightarrow\left(z_{1}+\frac{1}{2}, z_{2}+\frac{1}{2}, z_{3}+\frac{1}{2}\right)
$$

produces again fixed tori from the three twisted sectors $\alpha, \beta$ and $\alpha \beta$. Under the action of the $\gamma$-shift, the fixed tori from each twisted sector are paired. Therefore, $\gamma$ reduces the total number of fixed tori from the twisted sectors by a factor of 2 , yielding $\left(h_{11}, h_{21}\right)=(27,3)$. This model therefore reproduces the data of the $Z_{2} \times Z_{2}$ orbifold at the free-fermion point in the Narain moduli space. The precise form of the shift that reproduces the $Z_{2} \times Z_{2}$ orbifold at the free fermionic model is given in [39], and differ slightly from [5]. However, these two models are in the same moduli space, and hence are connected by continuous extrapolations. Eq. (5) suffices for our discussion here.

Despite its innocuous appearance the connection between $X_{1}$ and $X_{2}$ by a freely acting shift has an important consequence. From the Standard Model data we may hypothesize that any realistic string vacuum should possess at least two ingredients. First, it should contain three chiral generations, and second, it 
should admit their $\mathrm{SO}(10)$ embedding. This $\mathrm{SO}(10)$ embedding is not realized in the low energy effective field theory limit of the string models, but is broken directly at the string level. The main phenomenological implication of this embedding is that the weak-hypercharge has the canonical GUT embedding.

It has been argued that the $Z_{2} \times Z_{2}$ orbifold naturally gives rise to three chiral generations. The reason being that it divides the six dimensional compactified manifold into three cyclicly symmetric spaces. It contains three twisted sectors and each of these sectors produces one chiral generation. The existence of exactly three twisted sectors arises, essentially, because we are modding out a three dimensional complex manifold, or a six dimensional real manifold, by $Z_{2}$ projections, which preserve the holomorphic three form. Thus, metaphorically speaking, the reason being that six divided by two equals three.

However, this argument holds for any $Z_{2} \times Z_{2}$ orbifold of a six dimensional compactified space, and in particular it holds for the $X_{1}$ manifold. Therefore, we can envision that this manifold can produce, in principle, models with $\mathrm{SO}(10)$ gauge symmetry, and three chiral generations from the three twisted sectors. However, the caveat is that this manifold is simply connected and hence the $\mathrm{SO}(10)$ symmetry cannot be broken by the Hosotani-Wilson symmetry breaking mechanism [40. The consequence of adding the freely acting shift (5) is that the new manifold $X_{2}$, while still admitting three twisted sectors is not simply connected and hence allows the breaking of the $\mathrm{SO}(10)$ symmetry to one of its subgroups.

The freely acting shift has the crucial function of connecting between the simply connected covering manifold to the non-simply connected manifold. Precisely such a construction has been utilized in 41] to construct non-perturbative vacua of heterotic M-theory. In the next section I discuss these phenomenological aspects of M-theory.

\section{M-embeddings}

The profound new understanding of string theory that emerged over the past few years means that we can use any of the perturbative string limits, as well as eleven dimensional supergravity to probe the properties of the fundamental M-theory vacuum. The pivotal property that this vacuum should preserve is the $S O(10)$ embedding of the Standard Model spectrum. This inference follows from the fact that also in the strong coupling limit heterotic M-theory produces discrete matter and gauge representations. Additionally, the underlying compactification should allow for the breaking of the $S O(10)$ gauge symmetry. In string theory the prevalent method to break the $S O(10)$ gauge group is by utilizing Wilson line symmetry breaking. Compactification of M-theory on manifolds with $S U(5)$ GUT gauge group that can broken to the Standard Model gauge group were discussed in 41. In 42 the analysis was extended to $S O(10)$ GUT gauge group that can be broken to $S U(5) \times U(1)$. This work was reviewed in 13 . and here I discuss relevant points for further explorations of the phenomenological free fermionic models. 
The key to the construction of ref. 41] is the utilization of elliptically fibered Calabi-Yau threefolds. These manifolds are represented as a two dimensional complex base manifold and a one dimensional complex fiber with a section. On these manifolds the equation for the fiber is given in the Weierstrass form $y^{2}=x^{3}+f\left(z_{1}, z_{2}\right) x+g\left(z_{1}, z_{2}\right)=\left(x-e_{1}\right)\left(x-e_{2}\right)\left(x-e_{3}\right)$. Here $f$ and $g$ are polynomials of degrees 8 and 12 , respectively and are functions of the base coordinates; $e_{1}, e_{2}$ and $e_{3}$ are the three roots of the cubic equation. Whenever two of the roots coincide the fiber degenerates into a sphere. Thus, there is a locus of singular fibers on the base manifold. These singularities are resolved by splitting the fiber into two spherical classes $F$ and $F-N$. One being the original fiber minus the singular locus, and the second being the resolving sphere.

A nonperturbative vacuum state of the heterotic M-GUT-theory on the observable sector is specified by a set of $\mathrm{M}$-theory 5-branes wrapping a holomorphic 2 -cycle on the 3 -fold. The 5 -branes are described by a 4 -form cohomology class $[W]$ satisfying the anomaly-cancellation condition. This class is Poincaré-dual to an effective cohomology class in $H_{2}(X, \mathbf{Z})$ that can be written as $[W]=c_{2}(T X)-c_{2}\left(V_{1}\right)-c_{2}\left(V_{2}\right)=\sigma_{*}(w)+c(F-N)+d N$, where $c_{2}(T X)$, $c_{2}\left(V_{1}\right)$ and $c_{2}\left(V_{2}\right)$ are the second Chern classes of the tangent bundle and the two gauge bundles on the fixed planes; $c, d$ are positive definite integers, $\omega$ is a class in $B$, and $\sigma_{*}(\omega)$ is its pushforward to $X$ under $\sigma$.

The key to the M-theory embedding of the free fermionic models is their correspondence with the $Z_{2} \times Z_{2}$ orbifold. The starting point toward this end is the $X_{1}$ embedding manifold with $\left(h_{11}, h_{21}\right)=(51,3)$. The manifold is then rendered non-simply connected by the freely acting involution and the methodology of ref. 4142 can be adopted to construct viable M-theory vacua. The difference however is that now the fiber is more singular than the ones previously considered. The fiber of $X_{1}$ in Weierstrass form is given by $y^{2}=x^{3}+$ $f_{8}(w, \tilde{w}) x z^{4}+g_{12}(w, \tilde{w}) z^{6}$, where $f_{8}=\eta-3 h^{2}$, and $g_{12}=h\left(\eta-2 h^{2}\right), h=$ $K \prod_{i, j=1}^{4}\left(w-w_{i}\right)\left(\tilde{w}-\tilde{w}_{j}\right)$ and $\eta=C \prod_{i, j=1}^{4}\left(w-w_{i}\right)^{2}\left(\tilde{w}-\tilde{w}_{j}\right)^{2}$. Taking $w \rightarrow w_{i}$ $\left(\right.$ or $\left.\tilde{w} \rightarrow \tilde{w}_{i}\right)$ we have a $D_{4}$ singular fiber. These $D_{4}$ singularities intersect in 16 points, $\left(w_{i}, \tilde{w}_{j}\right), i, j=1, \ldots 4$, in the base. The resolution of the singular fiber in this case is more involved than the simpler ones previously considered. It is expected that the richer structure of fiber classes will yield a richer class of M-theory vacua with the possibility of new features appearing.

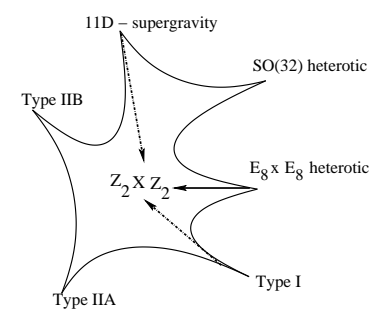

Fig. 1. Phenomenological application of M-theory 
Figure (11) illustrates qualitatively the approach to the phenomenological application of M-theory advocated in this paper. In this view the different perturbative M-theory limits are used to probe the properties of a specific class of compactifications. In this respect one may regard the free fermionic models as illustrative examples. Namely, in the heterotic limit this formulation highlighted the particular class of models that are connected to the $Z_{2} \times Z_{2}$ orbifold. In order to utilize the $\mathrm{M}$-theory advances to phenomenological purposes, our task then is to now explore the compactification of the other perturbative string limits on the same class of spaces, with the aim of gaining further insight into their properties. In this spirit compactifications of type I string theory on the $Z_{2} \times Z_{2}$ orbifold that are connected to the free fermionic models have been explored [43].

\section{On the origin of the three generations}

The free fermionic models correspond to $Z_{2} \times Z_{2}$ orbifold at an enhanced symmetry point in the Narain moduli space. As argued above the $Z_{2} \times Z_{2}$ orbifold, via its free fermion realization, naturally produces three generation models arising from the three twisted sectors. However, the geometrical correspondence of the free fermionic models is so far understood for the extended NAHE set models, i.e. for the case of the $X_{2}$ manifold with 24 generations. Hence, in order to promote the geometrical understanding of the origin of the three generations in the free fermionic models, it is important to understand the geometrical interpretation of the boundary condition basis vectors beyond the NAHE set.

Let us review for this purpose the vacuum structure in the twisted sectors $b_{1}, b_{2}$ and $b_{3}$. In the light-cone gauge the world-sheet free fermion field content includes: in the left-moving sector the two space-time fermions $\psi_{1,2}^{\mu}$ and the six real triples $\left\{\chi_{i}, y_{i}, \omega_{i}\right\}(i=1, \cdots, 6)$; in the right-moving sector the six real doubles $\left\{\bar{y}_{i}, \bar{\omega}_{i}\right\} \quad(i=1, \cdots, 6)$ and the sixteen complex fermions $\left\{\bar{\Psi}^{1, \cdots, 5}, \bar{\eta}^{1,2,3}, \bar{\phi}^{1, \cdots, 8}\right\}$. For our purpose the important set is the set of internal real fermions $\{y, \omega \mid \bar{y}, \bar{\omega}\}^{1, \cdots, 6}$. We can bosonize the fermions in this set by defining

$$
\mathrm{e}^{i X_{i}}=\frac{1}{\sqrt{2}}\left(y_{i}+i \omega_{i}\right) \quad \mathrm{e}^{i \bar{X}_{i}}=\frac{1}{\sqrt{2}}\left(\bar{y}_{i}+i \bar{\omega}_{i}\right)
$$

We recall that the vacuum of the sectors $b_{i}$ is made of 12 periodic complex fermions, $f$, each producing a doubly degenerate vacua $|+\rangle,|-\rangle$, annihilated by the zero modes $f_{0}$ and $f_{0}{ }^{*}$ and with fermion numbers $F(f)=0,-1$, respectively. The total number of states in each of these sector is therefore

$$
2^{12}=\sum_{n=0}^{12}\left(\begin{array}{c}
12 \\
n
\end{array}\right) .
$$

After applying the GSO projections the degeneracy at the level of the extended NAHE model distributes as follows:

$$
[y, \omega \mid \bar{y}, \bar{\omega}]_{b_{j}}\left(\psi^{\mu}, \chi_{j}\right) \quad\left[\bar{\psi}^{1, \cdots, 5}\right] \quad\left(\bar{\eta}_{j}\right)
$$




$$
\begin{aligned}
& {\left[\left(\begin{array}{l}
4 \\
0
\end{array}\right)+\left(\begin{array}{l}
4 \\
2
\end{array}\right)+\left(\begin{array}{l}
4 \\
4
\end{array}\right)\right] }\left\{\left(\begin{array}{l}
2 \\
0
\end{array}\right)\left[\left(\begin{array}{l}
5 \\
0
\end{array}\right)+\left(\begin{array}{l}
5 \\
2
\end{array}\right)+\left(\begin{array}{l}
5 \\
4
\end{array}\right)\right]\left(\begin{array}{l}
1 \\
0
\end{array}\right)\right. \\
&\left.+\left(\begin{array}{l}
2 \\
2
\end{array}\right)\left[\left(\begin{array}{l}
5 \\
1
\end{array}\right)+\left(\begin{array}{l}
5 \\
3
\end{array}\right)+\left(\begin{array}{l}
5 \\
5
\end{array}\right)\right]\left(\begin{array}{l}
1 \\
1
\end{array}\right)\right\}
\end{aligned}
$$

where $4=\left\{y^{3} y^{4}, y^{5} y^{6}, \bar{y}^{3} \bar{y}^{4}, \bar{y}^{5} \bar{y}^{6}\right\}, 2=\left\{\psi^{\mu}, \chi^{12}\right\}, 5=\left\{\bar{\psi}^{1, \cdots, 5}\right\}$ and $1=\left\{\bar{\eta}^{1}\right\}$. The combinatorial factor counts the number of $|-\rangle$ in a given state. The two terms in the curly brackets correspond to the two components of a Weyl spinor. The $10+1$ in the 27 of $E_{6}$ are obtained from the sector $b_{j}+\xi_{1}$. The states which count the multiplicities of $E_{6}$ are the internal fermionic states $\left\{y^{3, \cdots, 6} \mid \bar{y}^{3, \cdots, 6}\right\}$. A similar result is obtained for the sectors $b_{2}$ and $b_{3}$ with $\left\{y^{1,2}, \omega^{5,6} \mid \bar{y}^{1,2}, \bar{\omega}^{5,6}\right\}$ and $\left\{\omega^{1, \cdots, 4} \mid \bar{\omega}^{1, \cdots, 4}\right\}$ respectively, which suggests that these twelve states correspond to a six dimensional compactified orbifold with Euler characteristic equal to 48 .

The construction of the free fermionic models beyond the NAHE-set entails the construction of additional boundary condition basis vectors and the associated one-loop GSO phases. Their function is to reduce the number of generations and at the same time break the four dimensional gauge group. In terms of the former the reduction is primarily by the action on the set of internal world-sheet fermions $\{y, \omega \mid \bar{y}, \bar{\omega}\}$. As elaborated in the next section this set corresponds to the internal compactified manifold and the action of the additional boundary condition basis vectors on this set also breaks the gauge symmetries from the internal lattice enhancement. The later is obtained by the action on the gauge degrees of freedom which correspond to the world-sheet fermions $\left\{\bar{\psi}^{1, \cdots, 5}, \bar{\eta}^{1, \cdots, 3}, \bar{\phi}^{1, \cdots, 8}\right\}$. In the bosonic formulation this would correspond to Wilson-line breaking of the gauge symmetries, hence for the purpose of the reduction of the number of generations we can focus on the assignment to the internal world-sheet fermions $\{y, \omega \mid \bar{y}, \bar{\omega}\}$.

We can therefore examine basis vectors that do not break the gauge symmetries further, i.e. basis vectors of the form $b_{j}$, with

$$
\left\{\psi_{1,2}^{\mu} \chi_{j, j+1},(y, \omega \mid \bar{y}, \bar{\omega}), \bar{\psi}^{1, \cdots, 5}, \bar{\eta}_{j}\right\}=1
$$

for some selection of $(y, \omega \mid \bar{y}, \bar{\omega})=1$ assignments such that the additional vectors $b_{j}$ produce massless $S O(10)$ spinorials. We will refer to such vectors as spinorial vectors. The additional basis vectors $b_{j}$ can then produce chiral, or non-chiral, spectrum. The condition that the spectrum from a given such sector $b_{j}$ be chiral is that there exist another spinorial vector, $b_{i}$, in the additive group $\Xi$, such that the overlap between the periodic fermions of the internal set $(y, \omega \mid \bar{y}, \bar{\omega})$ is empty, i.e.

$$
\left\{b_{j}(y, \omega \mid \bar{y}, \bar{\omega})\right\} \cap\left\{b_{i}(y, \omega \mid \bar{y}, \bar{\omega})\right\} \equiv \emptyset .
$$

If there exists such a vector $b_{i}$ in the additive group then it will induce a GSO projection that will select the chiral states from the sector $b_{j}$. Interchangeably, if such a vector does not exist, the states from the sector $b_{j}$ will be non-chiral, i.e. there will be an equal number of 16 and $\overline{16}$ or 27 and $\overline{27}$. For example, we note that for the NAHE-set basis vectors the condition (17) is satisfied. Below 
I discuss the orbifold correspondence of this condition. The reduction to three generations in a specific model is illustrated in table 8

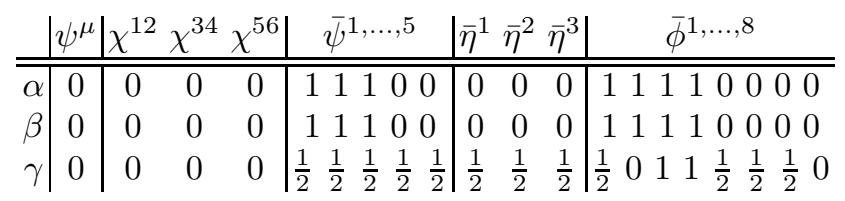

\begin{tabular}{c|cccc|cccc|cccc}
\multicolumn{1}{|c}{$y^{3} y^{6}$} & $y^{4} \bar{y}^{4}$ & $y^{5} \bar{y}^{5}$ & $\bar{y}^{3} \bar{y}^{6}$ & $y^{1} \omega^{5}$ & $y^{2} \bar{y}^{2}$ & $\omega^{6} \bar{\omega}^{6}$ & $\bar{y}^{1} \bar{\omega}^{5}$ & $\omega^{2} \omega^{4}$ & $\omega^{1} \bar{\omega}^{1}$ & $\omega^{3} \bar{\omega}^{3}$ & $\bar{\omega}^{2} \bar{\omega}^{4}$ \\
\hline \hline$\alpha$ & 1 & 0 & 0 & 0 & 0 & 0 & 1 & 1 & 0 & 0 & 1 & 1 \\
$\beta$ & 0 & 0 & 1 & 1 & 1 & 0 & 0 & 0 & 0 & 1 & 0 & 1 \\
$\gamma$ & 0 & 1 & 0 & 1 & 0 & 1 & 0 & 1 & 1 & 0 & 0 & 0
\end{tabular}

In the realistic free fermionic models the vector $X$ is replaced by the vector $2 \gamma$ in which $\left\{\bar{\psi}^{1, \cdots, 5}, \bar{\eta}^{1}, \bar{\eta}^{2}, \bar{\eta}^{3}, \bar{\phi}^{1, \cdots, 4}\right\}$ are periodic. This reflects the fact that these models have $(2,0)$ rather than $(2,2)$ world-sheet supersymmetry. At the level of the NAHE set we have 48 generations. One half of the generations is projected because of the vector $2 \gamma$. Each of the three vectors in table 8 acts nontrivially on the degenerate vacuum of the fermionic states $\{y, \omega \mid \bar{y}, \bar{\omega}\}$ that are periodic in the sectors $b_{1}, b_{2}$ and $b_{3}$ and reduces the combinatorial factor of Eq. (6) by a half. Thus, we obtain one generation from each sector $b_{1}, b_{2}$ and $b_{3}$.

The geometrical interpretation of the basis vectors beyond the NAHE set is facilitated by taking combinations of the basis vectors in 8 which entails choosing another set to generate the same vacuum. The combinations $\alpha+\beta$, $\alpha+\gamma, \alpha+\beta+\gamma$ produce the following boundary conditions under the set of internal real fermions

\begin{tabular}{c|cccc|ccccc|ccccc} 
& \multicolumn{3}{|c}{$y^{3} y^{6}$} & $y^{4} \bar{y}^{4}$ & $y^{5} \bar{y}^{5}$ & $\bar{y}^{3} \bar{y}^{6}$ & $y^{1} \omega^{5}$ & $y^{2} \bar{y}^{2}$ & $\omega^{6} \bar{\omega}^{6}$ & $\bar{y}^{1} \bar{\omega}^{5}$ & $\omega^{2} \omega^{4} \omega^{1} \bar{\omega}^{1} \omega^{3} \bar{\omega}^{3} \bar{\omega}^{2} \bar{\omega}^{4}$ \\
\hline \hline$\alpha+\beta$ & 1 & 0 & 1 & 1 & 1 & 0 & 1 & 1 & 0 & 1 & 1 & 0 & \\
$\beta+\gamma$ & 0 & 1 & 1 & 0 & 1 & 1 & 0 & 1 & 1 & 1 & 0 & 1 & $(9)$ \\
$\alpha+\beta+\gamma$ & 1 & 1 & 1 & 0 & 1 & 1 & 1 & 0 & 1 & 1 & 1 & 0
\end{tabular}

It is noted that the two combinations $\alpha+\beta$ and $\beta+\gamma$ are fully symmetric between the left and right movers, whereas the third, $\alpha+\beta+\gamma$, is fully asymmetric. The action of the first two combinations on the compactified bosonic coordinates translates therefore to symmetric shifts. Thus, we see that reduction of the number of generations is obtained by further action of fully symmetric shifts.

Due to the presence of the third combination the situation, however, is more complicated. The third combination in 9 is fully asymmetric between the left and right movers and therefore does not have an obvious geometrical interpretation. In fact, a complete classification of all the possible $Z_{2} \times Z_{2}$ orbifold models with symmetric shifts on complex tori, reveals that three generations are not obtained 
44. Three generations are obtained in the free fermionic models by the inclusion of the asymmetric shift. This observation has profound implications on the type of geometries that are related to the realistic string vacua, as well as on the issue of moduli stabilization.

The same conclusion can also be obtained by using purely bosonic language. Starting with three complex tori parameterized by three complex coordinates, the torus identification is given by (3). The symmetric shift action is

$$
z_{i}=z_{i}+\frac{1}{2} \quad \text { and } \quad z_{i}=z_{i}+\frac{\tau}{2}
$$

and a given action may act on any number of the three tori. The additional shifts may have the following actions:

$$
\begin{array}{ll}
\text { freely acting } & \longrightarrow \oplus\left(h_{11}=h_{21}=0\right) \\
\text { chiral preserving } & \longrightarrow \oplus\left(h_{11}=h_{21}\right) \\
\text { non freely acting } & \longrightarrow \oplus\left(h_{11} \neq h_{21}\right)
\end{array}
$$

In the first case one of the tori is always shifted and hence there are no fixed points and the action is free. In the second case we have tori above fixed points and all the other geometrical identifications preserve the fixed tori. Since the contribution of $T_{2}$ gives $\oplus h_{11}=\oplus h_{21}=1$ we have that this case preserves the chirality. In the third case we have a situation that for a fixed torus we impose the identification $z_{k} \leftrightarrow-z_{k}$. In this case the torus above the fixed point degenerates to $P_{1}$, for which $\oplus h_{11}=1, \oplus h_{21}=0$ and therefore this case adds to the net chirality. In ref. [45] we have classified all the possible shifts on the three complex tori, and obtained the same result. Three generations are not possible for $Z_{2} \times Z_{2}$ orbifolds of three complex tori, with purely symmetric shifts.

\section{Neutrino mass textures}

The neutrino sector of the Standard Model provides another piece to the flavor enigma. Evidence for neutrino oscillations steadily accumulated over the past few years, resulting in compelling evidence for neutrino masses. This in turn points to the augmentation of the Standard Model by the right-handed neutrinos, and provides further evidence for the elegant embedding of the Standard Model matter states, generation by generation, in the 16 spinorial representation of $S O(10)$. However, in this respect the new neutrino data raises further puzzles. The observation of a zenith angle dependence of $\nu_{\mu}$ from cosmic ray showers at super-Kamiokande [2] provides strong evidence for oscillations in atmospheric neutrinos with maximal $\nu_{\mu} \rightarrow \nu_{\tau}$ oscillations, whereas the observations at the solar Sudbury Neutrino Observatory (SNO) 3] and at the reactor KamLAND experiment 4] favor the large mixing angle MSW solution of the solar neutrino problem 46. The recent data from the Wilkinson Microwave Anisotropy Probe (WMAP) on cosmic microwave background anisotropies 6], combined with the 2 degree Field Galaxy Redshift Survey, CBI and ACBAR [4], restricts the amount 
of critical density attributed to relativistic neutrinos, and imposes that the sum of the masses is smaller than $0.75 \mathrm{eV}$.

While the Standard Model data strongly supports the incorporation of the Standard Model gauge and matter spectrum in representations of larger gauge groups, the flavor sector of the Standard Model provides further challenges. In the heavy generation the consistency of the bottom-quark-tau lepton mass ratio with the experimental data arises due to the running of the strong gauge coupling. The remaining flavor data, however, must have its origin in a theory that incorporates gravity into the picture. Most developed in this context are the string theories that provide a viable perturbative framework for quantum gravity. However, a new twist of the puzzle arises due to the fact that while in the quark sector we observe an hierarchical mass pattern with suppressed mixing angles, the observations in the neutrino sector are compatible with large mixing angles that implies approximate mass degeneracy.

An elegant mechanism in the context of $S O(10)$ unification to explain the large mixing in the neutrino sector was proposed in ref. 48. However, this mechanism utilizes the 126 of $S O(10)$, that does not arise in perturbative string theories 49 . On the other hand, string constructions offer a solution to the proton longevity problem. A doublet-triplet splitting mechanism is induced when the $S O(10)$ symmetry is broken to $S O(6) \times S O(4)$ by Wilson-lines 32 . In the stringy doublet-triplet splitting mechanism the color triplets are projected from the massless spectrum and the doublets remain light. Additional symmetries that arise in the string models may also explain the suppression of proton decay from dimension four and gravity mediated operators. String constructions also explain the existence of three generations in terms of the geometry of the compactified manifold. It is therefore important to seek other explanations for the origin of the discrepancy in the quark and lepton mass sectors. An alternative possibility to the utilization of the 126 in the seesaw mechanism is to use the nonrenormalizable term $1616 \overline{1616}$. In this case the $B-L$ symmetry is broken along a supersymmetric flat direction by the VEVs of the neutral components of $\left\langle 16_{H}\right\rangle=\left\langle\overline{16}_{H}\right\rangle$, where $16_{H}$ and $\overline{16}_{H}$ are two Higgs multiplets, distinct from the three Standard Model generations. This term then induces the heavy Majorana mass term for the right-handed neutrino. The contemporary studies of neutrino masses in this context are based on this term. We will refer to this as the "one-step seesaw mechanism" [50]. Similarly, explorations in the context of type I string inspired models also use the "one-step seesaw mechanism" [51. In this talk we propose that the neutrino data points to the role of $S O(10)$ singlet fields in the see-saw mass matrix.

The two-step seesaw mechanism utilizes the $S O(10)$ singlets fields that are abundant in string models. An inspired version of this mechanism then takes the form

$$
\left(\begin{array}{ccc}
0 & M_{D} & 0 \\
M_{D} & 0 & M_{\chi} \\
0 & M_{\chi} & M_{\phi}
\end{array}\right)
$$


The left-handed Majorana mass matrix is given by

$$
M_{\nu}=M_{D} M_{\chi}^{-1} M_{\phi} M_{\chi}^{-1} M_{D}^{T} .
$$

The flavor structure of $m_{\nu_{L}}$ arises from $m_{\phi}$ and can therefore account for the left-handed neutrino large while not disturbing the small mixing in the quark sector. This mechanism however requires the $\phi_{i} S O(10)$ singlet fields to exist at intermediate energies that presents a $\mu_{\phi}$ problem 53 .

\section{Conclusions and outlook}

I discussed in this paper the enterprise of string phenomenology in light of LEP, KAMLAND and WMAP. These three experiments represent the reward of decades of dedicated experimental efforts by numerous people, who contribute anonymously to the accumulated scientific knowledge. Their efforts should be saluted and are humbly and gratefully acknowledged. Their results enable the theoretical pursuit of the proposition of unification, string phenomenology, Mtheory, and the like. The particle experiments suggest two key ingredients that a realistic vacuum should possess. The existence of three generations together with their grand unification embedding, most appealing in 16 spinorial representations of $S O(10)$. The free fermionic models admit these two pivotal components. It is of course not suggested that any of the contemporary three generation models is the right one. That indeed would be folly. Furthermore, it may also well be that at the end of the day the free fermionic construction will not suffice to describe the full details of the true vacuum. Nevertheless, it may still be the case that the fermionic models are able to extract some key properties of the true vacuum. It is then our task to try to isolate what those key properties may be. It is proposed that the realistic free fermionic models suggest two such pivotal properties. The first is the relation of the free fermionic point to the self-dual point under T-duality. The second is the fact that the $Z_{2}$ orbifold twistings that are utilized in the free fermionic models act on the coordinates as real coordinates. This is furthermore compatible with the necessity of introducing an asymmetric shift in the reduction to three generations. Such a reduction may imply that the complex structure of the Calabi-Yau threefold is necessarily broken. Hence providing phenomenological guideline to the class of geometries that should be sought to construct realistic string vacua. Furthermore, the asymmetric orbifold projection implies that some of the moduli must be fixed. Incorporation of these observations in the utilization of string dualities to study these vacua, may provide further insight into their properties. WMAP data paves the way for a new decade of exciting experimental data that may yet challenge our perception of physical reality. In the least it provides a more accurate account of the energy composition of the universe, that the true string vacuum should accommodate. The forthcoming years will provide further data with the UHECR experiments exploring the viability of top-down models. The first priority of these experiments is clearly to establish or refute the breaching of the GZK cutoff, but the possibility that they may provide signals for new physics were entertained in 
ref. [52. Finally, the LHC will explore the electroweak symmetry breaking sector, and subsequently the TLC (The Linear Collider) will be vitally needed to provide precision measurements.

\section{References}

1. See e.g. ALEPH, DELPHI, L3 and OPAL collaborations: Phys. Lett. B 565, 61 (2003)

2. Y. Fukuda et al [Super-Kamiokande Collaboration]: Phys. Rev. Lett. 81, 1562 (1998)

3. Q.R. Ahmad et al [SNO collaboration]: Phys. Rev. Lett. 89, 011301 (2002)

4. K. Eguchi et al [KamLAND collaboration]: Phys. Rev. Lett. 90, 021802 (2003)

5. J.C. Pati and A. Salam, Phys. Rev. D 10, 275 (1974)

6. C.L. Bennett et al: astro-ph/0302207, D.N. Spergel et al: astro-ph/0302209

7. For reviews and references see e.g.: P.K. Townsend: hep-th/9612121 A. Sen: hep-th/9802051 M.J. Duff: hep-th/9805177. B. Ovrut: hep-th/0201032

8. For review and references see e.g.: A. Giveon et al: Phys. Rep. 244, 77 (1994)

9. P. Ginsparg: Nucl. Phys. B 295, 153 (1988)

10. N. Seiberg and E. Witten: Nucl. Phys. B 426, 19 (1994)

11. A. Font et al: Phys. Lett. B 249, 35 (1990); S.J. Rey: Phys. Rev. D 43, 5261 (91); A. Sen: Phys. Lett. B 329, 217 (1994)

12. E. Witten: Nucl. Phys. B 443, 85 (1995)

13. A.E. Faraggi: hep-ph/9707311 hep-th/9910042 hep-th/0208125 hep-th/0307037

14. K. Kawai et al: Nucl. Phys. B 288, 1 (1987); I. Antoniadis et al: Nucl. Phys. B 289, 87 (1987)

15. A.E. Faraggi and D.V. Nanopoulos: Phys. Rev. D 48, 3288 (1993); A.E. Faraggi: hep-th/9511093 hep-th/9708112

16. I. Antoniadis et al: Phys. Lett. B 231, 65 (1989)

17. I. Antoniadis et al: Phys. Lett. B 245, 161 (1990); G.K. Leontaris and J. Rizos: Nucl. Phys. B 554, 3 (1999)

18. A.E. Faraggi et al: Nucl. Phys. B 335, 347 (1990); A.E. Faraggi: Phys. Rev. D 46, 3204 (1992)

19. A.E. Faraggi: Phys. Lett. B 278, 131 (1992); Nucl. Phys. B 387, 239 (1992)

20. A.E. Faraggi: Phys. Lett. B 274, 47 (1992); Phys. Lett. B 377, 43 (1995); Nucl. Phys. B 487, 55 (1996)

21. G.B. Cleaver et al: Phys. Lett. B 455, 135 (1999); Int. J. Mod. Phys. A 16, 425 (2001); Nucl. Phys. B 593, 471 (2001); Mod. Phys. Lett. A 15, 1191 (2000); Int. J. Mod. Phys. A 16, 3565 (2001); Nucl. Phys. B 620, 259 (2002)

22. G.B. Cleaver et al: Phys. Rev. D 63, 066001 (2001); Phys. Rev. D 65, 106003 (2002); hep-ph/0301037

23. S. Chang et al: Phys. Lett. B 397, 76 (1997); Nucl. Phys. B 477, 65 (1996); J. Elwood and A.E. Faraggi: Nucl. Phys. B 512, 42 (1998)

24. S. Chaudhuri et al: Nucl. Phys. B 469, 357 (1996);

25. G.B. Cleaver et al: Nucl. Phys. B 525, 3 (1998); Nucl. Phys. B 545, 47 (1998); Phys. Rev. D 59, 055005 (1999); Phys. Rev. D 59, 115003 (1999)

26. For review see e.g.: J. Lykken hep-ph/9511456 J.L. Lopez hep-ph/9601208 A.E. Faraggi hep-ph/9404210

27. F. Abe et al: Phys. Rev. Lett. 74, 2626 (1995); S. Abachi et al: Phys. Rev. Lett. 74, 2632 (1995) 
28. A.E. Faraggi: Nucl. Phys. B 403, 101 (1993);Nucl. Phys. B 407, 57 (1993)

29. I. Antoniadis et al: Phys. Lett. B 278, 257 (1992); A.E. Faraggi and E. Halyo: Phys. Lett. B 307, 305 (1993); Nucl. Phys. B 416, 63 (1994); J. Ellis et al: Phys. Lett. B 425, 86 (1998)

30. I. Antoniadis et al: Phys. Lett. B 279, 281 (1992); A.E. Faraggi and E. Halyo: Phys. Lett. B 307, 311 (1993); A.E. Faraggi and J.C. Pati: Phys. Lett. B 400, 314 (1997)

31. I. Antoniadis et al: Phys. Lett. B 268, 188 (1991); I. Antoniadis et al: Phys. Lett. B 272, 31 (1991); A.E. Faraggi: Phys. Lett. B 302, 202 (1993); K.R. Dienes and A.E. Faraggi: Phys. Rev. Lett. 75, 2646 (1995); Nucl. Phys. B 457, 409 (1995)

32. A.E. Faraggi: Nucl. Phys. B 428, 111 (1994); Phys. Lett. B 339, 223 (1994); Phys. Lett. B 398, 95 (1997); Phys. Lett. B 499, 147 (2001); Phys. Lett. B 520, 337 (2001); J.C. Pati: Phys. Lett. B 388, 532 (1996); J. Ellis et al: Phys. Lett. B 419, 123 (1998); A.E. Faraggi and M. Thormeier Nucl. Phys. B 624, 163 (2002)

33. I. Antoniadis et al: Phys. Lett. B 241, 24 (1990); A.E. Faraggi and E. Halyo: Int. J. Mod. Phys. A 11, 2357 (1996); A.E. Faraggi and J.C. Pati: Nucl. Phys. B 526, 21 (1998); A.E. Faraggi and O. Vives: Nucl. Phys. B 641, 93 (2002)

34. A. Dedes and A.E. Faraggi: Phys. Rev. D 62, 016010 (2000)

35. X.G. Wen and E. Witten: Nucl. Phys. B 261, 651 (1985); A. Schellekens: Phys. Lett. B 237, 363 (1990); J. Ellis et al: Phys. Lett. B 247, 257 (1990); J. Ellis et al: Nucl. Phys. B 373, 399 (1992); K. Benakli et al: Phys. Rev. D 59, 047301 (1999); S. Sarkar and R. Toldra: Nucl. Phys. B 621, 495 (2002)

36. A.E. Faraggi, K.A. Olive and M. Pospelov: Astrophys. J. 13, 31 (2000)

37. C. Corianò, A.E. Faraggi and M. Plumacher: Nucl. Phys. B 614, 233 (2001)

38. A.E. Faraggi: Phys. Lett. B 326, 62 (1994); P. Berglund et al: Phys. Lett. B 433, 269 (1998); Int. J. Mod. Phys. A 15, 1345 (2002)

39. A.E. Faraggi: Phys. Lett. B 544, 207 (2002)

40. Y. Hosotani: Phys. Lett. B 126, 309 (83); Phys. Lett. B 129, 193 (83)

41. R. Donagi et al: hep-th/9901009 hep-th/9912208 Class. Quant. Grav. 17, 1049 (2000)

42. A.E. Faraggi et al: Nucl. Phys. B 641, 111 (2002); A.E. Faraggi and R. Garavuso: Nucl. Phys. B 659, 224 (2003)

43. D.J. Clements and A.E. Faraggi: hep-th/0302006

44. A.E. Faraggi, C. Kounnas, S.E.M. Nooij and J. Rizos, hep-th/0311058 and paper in preparation

45. A.E. Faraggi and R. Donagi: paper in preparation

46. L. Wolfenstein: Phys. Rev. D 17, 2369 (1978); S.P. Mikheev and A.Y. Smirnov: Sov. J. Nucl. Phys. 42, 913 (1985)

47. O. Elgaroy et al: Phys. Rev. Lett. 89, 38 (2002); T.J. Pearson et al: astro-ph/0205388 C.I. Kuo et al: astro-ph/0212289

48. B. Bajc, G. Senjanovic and F. Vissani: Phys. Rev. Lett. 90, 051802 (2003)

49. K.R. Dienes and J. March-Russell: Nucl. Phys. B 479, 113 (1996)

50. M. Gell-Mann, P.Ramond and R. Slansky, in Supergravity, ed. by D. Freedman and P. Van-Nieuwenhuizen (North-Holland 1979), p. 315; T. Yanagida, Proc. Workshop on "Unified Theories and Baryon Number of the Universe", eds. Sawata and A. Sugamoto, KEK, Japan (1979); R.N. Mohapatra and G. Senjanovic, Phys. Rev. Lett. 44, $912(80)$

51. See e.g. S.F. King: hep-ph/0208270 and references therein

52. A Cafarella et al: hep-ph/0306236 hep-ph/0308169 hep-ph/0309159

53. C. Corianò and A.E. Faraggi, Phys. Lett. B 581, 99 (2003). 